\title{
The Brain as Target Image Detector: The Role of Image Category and Presentation Time
}

\author{
Anne-Marie Brouwer ${ }^{1}$, Jan B.F. van $\operatorname{Erp}^{1}$, Bart Kappé ${ }^{1}$, and Anne E. Urai ${ }^{1,2}$ \\ ${ }^{1}$ TNO Human Factors, Kampweg 5, 3769 ZG Soesterberg, The Netherlands \\ ${ }^{2}$ University College Utrecht, P/O. Box 80145, 3508 TC Utrecht, The Netherlands \\ \{anne-marie.brouwer, jan.vanerp\}@tno.nl, bart.kappe@xs4all.nl, \\ anne.urai@gmail.com
}

\begin{abstract}
The brain can be very proficient in classifying images that are hard for computer algorithms to deal with. Previous studies show that EEG can contribute to sorting shortly presented images in targets and non-targets. We examine how EEG and classification performance are affected by image presentation time and the kind of target: humans (a familiar category) or kangaroos (unfamiliar). Humans are much easier detected as indicated by behavioral data, EEG and classifier performance. Presentation of humans is reflected in the EEG even if observers were attending to kangaroos. In general, $50 \mathrm{~ms}$ presentation time decreased markers of detection compared to $100 \mathrm{~ms}$.
\end{abstract}

\section{Introduction}

Recent technological developments have lowered the costs of gathering and storing high volumes of images. Enormous amounts of images are digitally available in fields ranging from internet search engines to security cameras and satellite streams. Finding an image of interest requires a system of image triage through which only a subset of images is selected for further visual inspection. However, in some cases, automatic analysis of image contents is difficult because computer vision systems lack the sensitivity, specificity and generalization skills needed for efficient image triage. The human brain, on the other hand, can be extremely apt at image classification and can recognize target images quickly and precisely. Participants in a study by Thorpe et al. [1] had to indicate whether a previously unseen photograph, flashed for just $20 \mathrm{~ms}$, contained an animal or not by releasing or holding a button. Already $150 \mathrm{~ms}$ after stimulus onset EEG (electroencephalography) signals for target and non-targets started to differ reliably- a frontal negativity developed for non-target images. Similar results were found by Goffaux et al. [2] where observers had to categorize types of landscape. An image classification BCI (Brain Computer Interface) may provide us access to these very powerful brain mechanisms to interpret images and enable observers to reliably classify images at very high speeds.

Several groups have already implemented image classification BCIs, usually based on a particular event related potential (ERP) present in the EEG, called the P3. The P3 is a positive peak in EEG that occurs approximately $300 \mathrm{~ms}$ after a target stimulus (a stimulus that the observer is attending to) is presented [3]. Sajda, Parra, Gerson and colleagues [4-7] presented their observers with sequences of 50 to 100 images of 
natural scenes, where each image was presented for $100 \mathrm{~ms}$. Observers had to press or release a button right after detecting a natural scene containing people, or after the sequence had ended. Each sequence contained 1 or 2 of these targets. They found that both EEG and button presses contributed to correctly ordering images from more to less likely to be a target. Similarly, Huang, Pavel, and colleagues [8-10] presented sequences of 50 satellite images, where each image was presented for 60 to $200 \mathrm{~ms}$. Targets were man-made objects such as ships, oil storage depots or golf courses. Half of the sequences contained 1 target, the other half none. Observers pressed a button directly after detecting the target or after the sequence had ended. They also found that both EEG and button presses contributed to correct classification.

The previous studies show the feasibility of image classification BCIs. In our research we want to build a BCI to classify shortly presented images, but in line with virtually all real-life image classification situations and (partly) in contrast to the previous studies, observers are unaware of the number of targets. This may be an important factor. If observers know that one target will be present, they may quit paying attention after target detection, or, if they did not see the target yet, anticipate it towards the end of the sequence. Also note that few compared to many targets may enhance P3 size [3]. We here focus on the role of the image category of the target, or target type, within a fixed collection of context images. It may not be possible to generalize results of the studies mentioned before when other types of targets (within other types of contexts) are searched. When e.g. looking for a human in a natural environment, the observers' expertise of human appearances can support performance. In this study we compare brain responses to attended or unattended images of humans to those of kangaroos. Thus, we compare between groups of images that are always the same, the only difference being which group attention is focused on. Since our European observers are more familiar with recognizing humans than kangaroos, detecting humans amongst other animals may be easier than detecting kangaroos and correspondingly, produce stronger P3s. In addition, specific ERP components that are associated with faces or other highly familiar stimuli such as the N170 may be present [11-13]. If so, and if in a particular image classification case the target of interest corresponds to such a familiar stimulus, these could be used in classifiers. Together with the effect of target type, we examine the effect of presentation time (100 or $50 \mathrm{~ms})$. Interactions between target type and presentation time may occur, such as kangaroo images eliciting P3s when they are presented long, but not when they are presented for a short time. Besides examining the ERPs directly, we also look for effects of target type and presentation time on classifier performance.

\section{Methods}

\subsection{Participants}

Twenty observers (10 men and 10 women) participated in the experiment. Their mean age was 38.9 years $(\mathrm{SD}=16.6)$. As verified by a questionnaire, all participants were neurologically healthy and had normal or corrected to normal vision. Participants gave their informed consent before the start of the experiment and were given a monetary reward for their time. 


\subsection{Stimuli}

All images were obtained from the Caltech-256 Object Category Dataset [14]. Images that were not clearly recognizable or had written text on them were excluded from the experiment. Only images in portrait format were used. In total 952 images were selected for use in the experiment, including 55 images of humans and 40 images of kangaroos. Images were normalized in size and in luminance using Matlab. Their size was reduced to $280 \times 420$ pixels. They were then transformed to the CIELAB Lab color space, where the average and standard deviation of luminance (L-component) were set to 30 and 25.2 respectively. Then, the images were transformed back to sRGB. Custom built software presented sequence of 60 images on a Dell 1907 LCD flat panel display (19 inch, $60 \mathrm{~Hz}, 1280 \times 1024$ pixels) at a viewing distance of about $70 \mathrm{~cm}$. Each image was presented for 50 or $100 \mathrm{~ms}$. In between image sequences, a white screen was shown for $1 \mathrm{~s}$ followed by a white screen with a black fixation cross that was presented for a randomly chosen interval between 0.8 and $1.2 \mathrm{~s}$.

\subsection{Design}

For each presentation time (50 and $100 \mathrm{~ms}$ ), each participant completed 10 runs with target type human and 10 runs with target type kangaroo. Each run consisted of 5 image sequences of 60 images each. Sequences could contain between 0 and 4 targets as well as 0 to 4 non-targets. Non-targets were images of kangaroos for the human target type and images of humans for the kangaroo target type. The resulting 25 combinations of target and non-target numbers were randomly distributed across runs and occurred twice within each of the four conditions (four combinations of target type and presentation time). Image sequences were generated taking into account the following constraints. There were always at least six fillers (images of animals that were neither humans nor kangaroos) between targets and non-targets. Targets and non-targets were never among the first or last 4 images. Within one run, images were never shown more than once. Half of the participants first performed the task at 100 $\mathrm{ms} / \mathrm{image}$ and then at $50 \mathrm{~ms} / \mathrm{image}$, the other half the other way around. The order of target types was counterbalanced across participants. The 10 runs were order balanced using a latin square.

\subsection{Task and Procedure}

Participants were seated comfortably in front of a monitor in a shielded room. Before the experiment started, the complete procedure was explained to the participants. The participants were asked to blink as little as possible and to limit any other movements during image sequences. The task was to concentrate on target images and count the number of times they were presented in an image sequence. The participants were informed of the nature of the targets (either humans or kangaroos) before every new target type. Every time that the target type and/or presentation speed changed, participants were given a training run to get used to the target type they had to detect and the speed of presentation. Participants entered the number of targets they had counted on a keypad during a time window of about $2 \mathrm{~s}$ in between image sequences. 


\subsection{EEG Recording}

EEG activity was recorded at the Fpz, Fz, Fp1, Fp2, Cz, Pz, P3, P4, Oz, POz, PO7 and PO8 electrode sites of the 10-20 system [15] using Au electrodes mounted in an EEG cap (g.tec medical engineering $\mathrm{GmbH}$ ). A ground electrode was attached to the scalp at the AFz electrode site. The EEG electrodes were referenced to linked mastoid electrodes. The impedance of each electrode was below $5 \mathrm{k} \Omega$. Data were sampled with a frequency of $256 \mathrm{~Hz}$ and filtered before storage by a $0.1 \mathrm{~Hz}$ high pass-, a $60 \mathrm{~Hz}$ low pass- and a $50 \mathrm{~Hz}$ notch filter (USB Biosignal Amplifier, g.tec medical engineering $\mathrm{GmbH}$ ). Additional electrodes (Kendall Neonatal ECG electrodes from Tyco Healthcare Deutschland $\mathrm{GmbH}$ ) were positioned above and below the left eye, and close to the outer canthi of the eyes to monitor EOG (electro-oculography - blinks and eye movements). EOG electrodes were referenced to each other. Data recording was controlled by a combination of custom-built software and Matlab/Simulink tools.

\subsection{Analysis}

EEG Signal Analysis. The EEG/EOG data were processed using Brain Vision Analyzer 2.0 (BrainProducts). We started out with data from the interval between the first fixation cross to $2 \mathrm{~s}$ after the last image of the run. All EEG channels were automatically inspected for bad episodes, using standard settings of Brain Vision Analyzer. This identified most of the eye blinks, which mainly occurred in between image sequences in accordance with experimental instructions. Bad episodes were excluded from the analysis. The remaining data were manually inspected for further irregularities to remove all eye blinks and other artifacts from the data. EOG data were not further used. Segments were then selected starting at $200 \mathrm{~ms}$ before image onset and $600 \mathrm{~ms}$ after image onset. Since there were many more filler segments than target and non-target segments, only every fourth of the filler segments was used. Segments were baseline corrected using an interval of $200 \mathrm{~ms}$ to $0 \mathrm{~ms}$ before stimulus onset. Averages were calculated for targets, non-targets and fillers per participant and condition. Visual inspection of these averages revealed that the N170 component appeared between 100 and $350 \mathrm{~ms}$ after stimulus onset. The P3 component appeared between 300 and $550 \mathrm{~ms}$ after stimulus onset. The area in $\mu \mathrm{V}^{*} \mathrm{~ms}$ within these timeframes was taken as a measure for the magnitude of the respective components.

For further $\mathrm{P} 3$ analysis, we selected data recorded at $\mathrm{Pz}$ because $\mathrm{Pz}$ is known to be a good location for measuring P3 [16] and these indeed distinguished well between targets and non-targets. More specifically, all electrodes distinguish well between targets and non-targets except for Fpz, Fp1 and Fp2. At these locations, paired t-tests on P3s per participant, electrode, targets and non-targets do not indicate significant differences between targets and non-targets ( $\mathrm{p}$-values $>0.11$ ). For all other electrodes, p-values are $<0.01$.

For further N170 analysis, we selected data from P4 since in our study, these appeared to distinguish best between images of humans and kangaroos. When target type is human, the N170 is larger for targets (human) than non-targets (kangaroo) at all electrodes (paired t-tests on N170 per participant, electrode, target and non-target resulted in p-values $<0.03$ ). When target type is kangaroo, the $\mathrm{N} 170$ tends to be larger 
for non-targets (human) than targets (kangaroo) at all electrodes; significantly so for $\mathrm{Pz}\left(\mathrm{t}_{19}=2.60, \mathrm{p}=0.02\right), \mathrm{P} 3\left(\mathrm{t}_{19}=2.75, \mathrm{p}=0.01\right), \mathrm{P} 4\left(\mathrm{t}_{19}=2.88, \mathrm{p}<0.01\right)$ and $\mathrm{PO} 7\left(\mathrm{t}_{19}=2.21\right.$, $\mathrm{p}=0.04)$.

We computed $\mathrm{dP} 3$ as the difference between the P3 elicited by targets and the P3 elicited by non-targets, separately for participants and conditions. A positive dP3 reflects a larger P3 for targets than for non-targets. This is the part of the P3 that we are interested in and that we want to check for sensitivity to target type and presentation time. Similarly, we computed dN170 as the difference between the N170 elicited by human images and the N170 elicited by kangaroo images, separately for participants and conditions. A positive dN170 reflects a larger N170 for human images than for kangaroo images.

Classification. We performed a cross-validation using a type of discriminant analysis as described in [17] which, in short, works as follows. EEG data was segmented in 1 sec epochs, starting at stimulus onset. EEG segments were normalized such that their average value was zero. For each participant and each run, segments following target stimuli were averaged across all electrodes. Segments following filler and non-target stimuli were averaged likewise. This was done using all sequences minus one. The difference between the two average responses (target minus filler/non-target) or 'template' was multiplied by single segments from the sequence left out when constructing the template. If for a certain segment, the mean result exceeded a certain threshold, it was interpreted as a response to a target, and otherwise as a response to a filler/non-target. The threshold was chosen such that we had equal percentages of target responses that were wrongly classified as filler/non-target responses and filler/non-target responses wrongly classified as target responses. This prevented the problem of judging a classifier that labels all segments as filler/non-targets as a good classifier since it only makes an error about twice in a sequence. The percentage of wrongly identified responses that we get when applying the threshold as described above is termed Equal Error Rate (EER). It is a measure of classification performance, where an EER of 50 means that a target or a non-target/filler has a 50\% chance of not being identified as such, thus reflecting chance performance, and an EER of 0 means perfect performance.

Statistical analysis. Repeated measures ANOVAs were used to test for effects of target type (human or kangaroo) and presentation time (50 or $100 \mathrm{~ms}$ ) on dP3, dN170, EER and counting error. Counting error was the absolute difference between the number of targets present in an image sequence and the number as reported by the participant. When appropriate, Tukey HSD post-hoc tests were performed to establish the nature of an effect. One sample t-tests were used to examine whether dP3 and dN170 were different from zero in all four conditions.

\section{Results}

\subsection{ERPs}

General. Figure 1 gives a general impression of the ERPs. It shows $\mathrm{Pz}$ grand averages of EEG separately for targets and non-targets, and for human and kangaroo 
images. Human targets appear to elicit both a negative component between 100 and $350 \mathrm{~ms}$ after stimulus onset (N170) and a positive component between 300 and $550 \mathrm{~ms}$ (P3). Non-target images of humans seem to elicit an N170 as well, but no P3. Images of kangaroos do not seem to generate a N170 or a P3, although the EEG seems slightly more positive for kangaroo targets than for kangaroo non-targets towards the end.

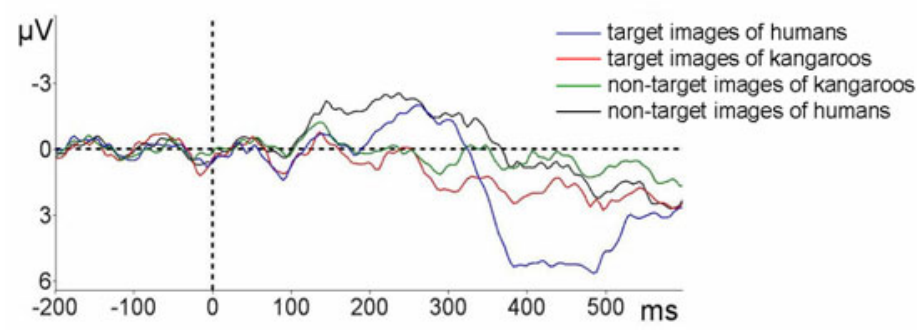

Fig. 1. EEG grand averages recorded at Pz, separately for targets and non-targets, and for human and kangaroo images. Time 0 corresponds to stimulus onset.

P3. Figure 2A shows dP3 ( $\mathrm{P} 3$ at $\mathrm{Pz}$ for the target minus that for the non-target) for each target type and presentation time. The mean values are larger than zero, except for the kangaroo target presented at $50 \mathrm{~ms}$, suggesting that generally an attention driven P3 is present. However, only for the human target presented at $100 \mathrm{~ms}$ the dP3 is significantly larger than 0 (one sample t-test $t_{19}=3.78, p<0.01$, other $p$-values $>0.32$ ). A repeated measures ANOVA indicates that the dP3 is affected by target type $\left(\mathrm{F}_{(1,19)}=5.61, \mathrm{p}=0.03\right)$ with the human target producing larger $\mathrm{dP} 3 \mathrm{~s}$ than the kangaroo, and presentation time $\left(\mathrm{F}_{(1,19)}=7.04, \mathrm{p}=0.02\right)$ with $100 \mathrm{~ms}$ producing larger $\mathrm{dP} 3 \mathrm{~s}$ than $50 \mathrm{~ms}$. There is no interaction between target type and presentation time $\left(\mathrm{F}_{(1,19)}=0.04\right.$, $\mathrm{p}=0.84)$.

N170. Figure $2 \mathrm{~B}$ shows $\mathrm{dN} 170$ (N170 at $\mathrm{P} 4$ for the human image minus that for the kangaroo) for each target type and presentation time. An N170 is present as indicated by mean dN170 values being significantly larger than zero for the human target types $\left(\mathrm{t}_{19}=3.08\right.$ for $100 \mathrm{~ms}$ presentation time and $\mathrm{t}_{19}=3.12$ for $50 \mathrm{~ms}$, both $\mathrm{p}$-values $\left.<0.01\right)$ and the kangaroo target type at $100 \mathrm{~ms}$ presentation time $\left(\mathrm{t}_{19}=3.37, \mathrm{p}<0.01\right)$. For the kangaroo type - $50 \mathrm{~ms}$ condition $\mathrm{dN} 170$ is not significantly larger than zero $\left(\mathrm{t}_{19}=0.34\right.$, $\mathrm{p}=0.98$ ). A repeated measures ANOVA indicates that there is no main effect of target type $\left(\mathrm{F}_{(1,19)}=0.01, \mathrm{p}=0.93\right)$ on $\mathrm{dN} 170$. There is an effect of presentation time $\left(\mathrm{F}_{(1,19)}=5.59, \mathrm{p}=0.03\right)$ and an interaction between target type and presentation time $\left(\mathrm{F}_{(1,19)}=6.36, \mathrm{p}=0.02\right)$. Tukey HSD post-hoc tests shows that for human target type conditions, presentation time does not affect the dN170 while for kangaroo target type conditions, dN170 is larger when presentation time is $100 \mathrm{~ms}$ compared to $50 \mathrm{~ms}$ (difference between the different presentation times for kangaroo target type conditions: $\mathrm{p}=0.02$; all other comparisons $\mathrm{p}$-values $>0.28$ ). 


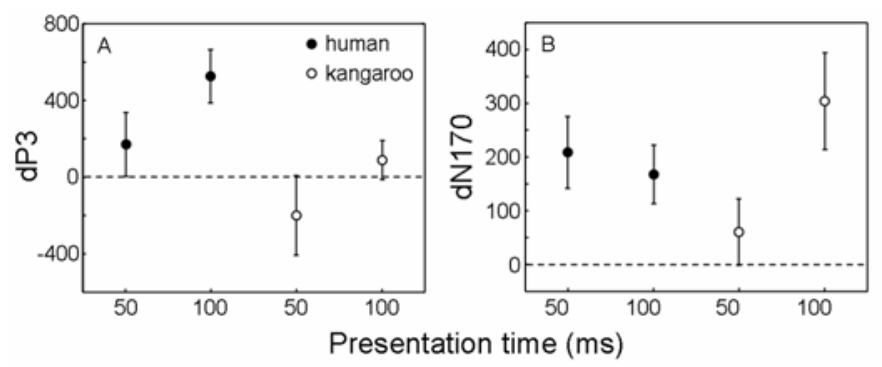

Fig. 2. $d P 3$ : $P 3$ at $P z$ for the target minus that for the non-target (A) and dN170: N170 at P4 for the human image minus that for the kangaroo (B) for each target type and presentation time. Error bars represent standard errors of the mean.

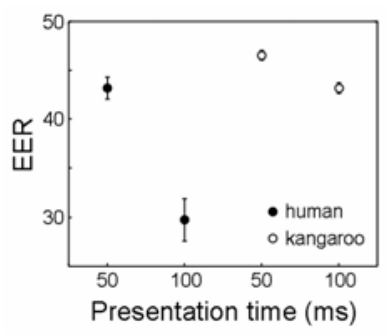

Fig. 3. Equal error rate for each target type and presentation time. Perfect classification performance would be at 0 and chance performance at 50 . Error bars represent standard errors of the mean.

\subsection{Classification}

Figure 3 shows mean equal error rate (EER) for each target type and presentation time where 0 indicates perfect classification performance and 50 chance performance. A repeated measures ANOVA showed an effect of presentation time $\left(\mathrm{F}_{(1,19)}=94.43\right.$, $\mathrm{p}<0.01)$, target type $\left(\mathrm{F}_{(1,19)}=36.69, \mathrm{p}<0.01\right)$ and an interaction $\left(\mathrm{F}_{(1,19)}=42.34, \mathrm{p}<0.01\right)$. Tukey HSD post-hoc tests indicated that the EER for human target at $100 \mathrm{~ms}$ was lower than the EER in all other conditions (p-values $<0.01$ for all comparisons) while there were no other significant differences between the EERs (p-values >0.11).

\subsection{Counting Error}

Figure 4A plots the number of targets reported against the number of targets presented, separately for each target type and presentation time. The figure shows a clear pattern of better performance for human compared to kangaroo targets, and for long compared to short presentation times. Better performance is reflected by stronger positive relations between counted and presented number of targets. Figure 4B shows the mean counting error for each target type and presentation time. Target type $\left(\mathrm{F}_{(1,19)}=405.00, \mathrm{p}<.01\right)$ and presentation time $\left(\mathrm{F}_{(1,19)}=75.39, \mathrm{p}<.01\right)$ both have an effect 
on counting error. There is an interaction between presentation time and target type $\left(\mathrm{F}_{(1,19)}=8.80, \mathrm{p}<.01\right)$ that indicates that counting human targets benefits more from a longer presentation time than kangaroo targets does.
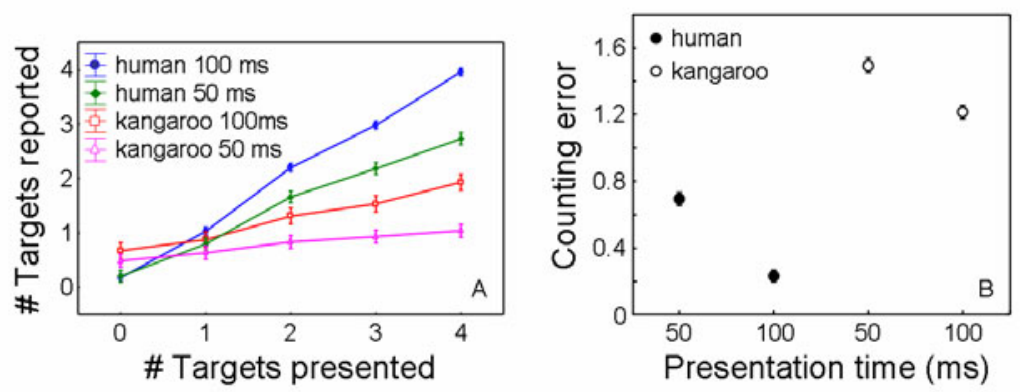

Fig. 4. Counting performance for each target type and presentation time as indicated by the number of targets reported against the number of targets presented (A) and counting error (B). Error bars represent $95 \%$ confidence intervals.

\section{Conclusions}

Our results clearly indicate that target type (in combination with the type of fillers) can affect performance of image classification BCIs. Images of humans as targets elicit stronger P3s than images of a less familiar species, kangaroos. In line with this, classification performance was better for human targets (when images were presented for $100 \mathrm{~ms}$ ) as well as counting performance. Detecting kangaroos in between animals appeared close to impossible with the short presentation times used and this was reflected in all dependent measures.

Human images also elicited an N170 whereas kangaroo images did not. For the presentation time of $100 \mathrm{~ms}$, this even held when observers focused on detecting images of kangaroos (in line with [18]). This means that besides the P3, the N170 can be used in classification algorithms when the goal of the image classification BCI is to find images of humans. Our finding that an electrode at the right hemisphere tended to display the N170 best is in line with previous findings (e.g. [19]). However, better N170 results are expected when EEG electrodes are placed more temporally than in the current study [20,21].

Besides target type, presentation time also affected the results. As demonstrated before, shorter presentation times (below $500 \mathrm{~ms}-$ [22]) result in smaller or harder to detect P3s and lower classification rates [4, 8, 23]. This is probably caused both by the fact that short presentation times limit processing time and because they increase overlap of ERPs resulting in noisier signals. Presentation time also interacted with target type. Presentation time did not affect the N170 with a human target, but when the target was a kangaroo, $100 \mathrm{~ms}$ presentation time produced a clearer N170 than 50 ms (in which case dN170 was not significant). For classification, longer presentation time increases performance, though not significantly so for the kangaroo, perhaps 
because this target type was just too difficult. Similarly, an interactive effect of presentation time and target type occurred for counting performance with longer presentation time increasing performance, but especially so for the human target.

In the present study, we focused on targets and non-targets, leaving ERPs elicited by the fillers largely aside. In general we can say that for the P3, filler and non-target data are virtually indistinguishable. The fact that non-targets do not elicit larger P3s than fillers, indicates that observers succeeded in switching attention between target types. Fillers elicit N170s that are in between those elicited by human images and kangaroo images. This is probably caused by filler images that resemble humans, like images of other primates. Note that the N170 would probably have been stronger when only images of human faces had been used instead of the broader category of images of humans that also included whole body pictures.

In conclusion, performance of image classification BCIs cannot simply be generalized to situations in which other types of targets are being searched. Image classification BCIs will probably be most successful when typical human expertise can be used, such as detecting human faces. In that case even unintentional detection could be tapped. In addition, future studies should take care not to design image classification BCIs for cases that can be effectively dealt with by computer algorithms.

Acknowledgements. The authors gratefully acknowledge the support of the BrainGain Smart Mix Programme of the Netherlands Ministry of Economic Affairs and the Netherlands Ministry of Education, Culture and Science, and David van Leeuwen for the classification analysis.

\section{References}

1. Thorpe, S., Fize, D., Marlot, C.: Speed of processing in the human visual system. Nature 381, 520-522 (1996)

2. Goffaux, V., Jacques, C., Mouraux, A., Oliva, A., Schyns, P.G., Rossion, B.: Diagnostic colours contribute to the early stages of scene categorization: Behavioural and neurophysiological evidence. Vis. Cogn. 12, 878-892 (2005)

3. Farwell, L.A., Donchin, E.: Talking off the top of your head: A mental prosthesis utilizing event-related brain potentials. Electroencephalography and Clinical Neurophysiology 70, 510-523 (1988)

4. Sajda, P., Gerson, A., Parra, L.: High-throughput image search via single-trial event classification in a rapid serial visual presentation task. In: Proc. First International IEEE EMBS Conference on Neural Engineering, pp. 7-10 (2003)

5. Gerson, A.D., Parra, L.C., Sajda, P.: Cortically-coupled Computer Vision for Rapid Image Search. IEEE Trans. on Neural Systems \& Rehabilitation Engineering 14, 174-179 (2006)

6. Sajda, P., Gerson, A.D., Philiastides, M.G., Parra, L.C.: Single-trial analysis of EEG during rapid visual discrimination: Enabling cortically-coupled computer vision. In: Dornhege, G., Mueller, K.-R. (eds.) Brain-Computer Interface. MIT Press, Cambridge (2007)

7. Parra, L.C., Christoforou, C., Gerson, A.D., Dyrholm, M., Luo, A., Wagner, M., Philiastides, M.G., Sajda, P.: Spatio-temporal linear decoding of brain state: Application to performance augmentation in high-throughput tasks. IEEE Signal Processing Magazine 25, 95-115 (2008) 
8. Huang, Y., Erdogmus, D., Mathan, S., Pavel, M.: Comparison of Linear and Nonlinear Approaches on Single Trial ERP Detection in Rapid Serial Visual Presentation Tasks. In: International Joint Conference on Neural Networks, pp. 1136-1142 (2006)

9. Huang, Y., Erdogmus, D., Mathan, S., Pavel, M.: A Fusion Approach for Image Triage using Single Trial ERP Detection. In: 3rd International IEEE/EMBS Conference on Neural Engineering, pp. 473-476 (2007)

10. Huang, Y., Erdogmus, D., Mathan, S., Pavel, M.: Large-scale image database triage via EEG evoked responses. In: IEEE International Conference on Acoustics, Speech and Signal Processing, pp. 429-432 (2008)

11. Bentin, S., McCarthy, G., Perez, E., Puce, A., Allison, T.: Electrophysiological studies of face perception in humans. J. Cogn. Neurosci. 8, 551-565 (1996)

12. Rossion, B., Jacques, C.: Does physical interstimulus variance account for early electrophysiological face sensitive responses in the human brain? Ten lessons on the N170. NeuroImage 39, 1959-1979 (2008)

13. Busey, T.A., Vanderkolk, J.R.: Behavioral and electrophysiological evidence for configural processing in fingerprint experts. Vis. Res. 45, 431-448 (2005)

14. Griffin, G., Holub, A., Perona, P.: Caltech-256 Object Category Dataset. California Institute of Technology (2007),

http: / / resolver. caltech. edu/Cal techAUTHORS: CNS-TR-2007-001

15. Jasper, H.: Report of the committee on methods of clinical examination in electroencephalography. Electroencephalography and Clinical Neurophysiology 10, 370375 (1958)

16. Ravden, D., Polich, J.: On P300 measurement stability: habituation, intra-trial block variation, and ultradian rhythms. Biol. Psych. 51, 59-76 (1999)

17. Bandt, C., Weymar, M., Samaga, D., Hamm, A.O.: A simple classification tool for singletrial analysis of ERP components. Psychophysiol. 46, 747-757 (2009)

18. Rousselet, G.A., Macé, M.J., Fabre-Thorpe, M.: Animal and human faces in natural scenes: How specific to human faces is the N170 ERP component? JOV 4, 13-21 (2004)

19. Rossion, B., Joyce, C.J., Cottrell, G.W., Tarr, M.J.: Early lateralization and orientation tuning for face, word and object processing in the visual cortex. Neuroimage 20, 16091624 (2003)

20. Kanwisher, N., McDermott, J., Chun, M.M.: The fusiform face area: A module in human extrastriate cortex specialized for face perception. J. Neurosci. 17(11), 4302-4311 (1997)

21. Luck, S.J.: An Introduction to the Event-Related Potential Technique. MIT Press, Cambridge (2005)

22. Shenoy, P., Tan, D.S.: Human-Aided Computing: Utilizing Implicit Human Processing to Classify Images. In: CHI 2008 Proceedings Cognition, Perception, and Memory (2008)

23. Yazdani, A., Vesin, J.-M., Izzo, D., Ampatzis, C., Ebrahimi, T.: Implicit retrieval of salient images using brain computer interface. In: Proceedings of International Conference on Image Processing, ICIP (2010) 